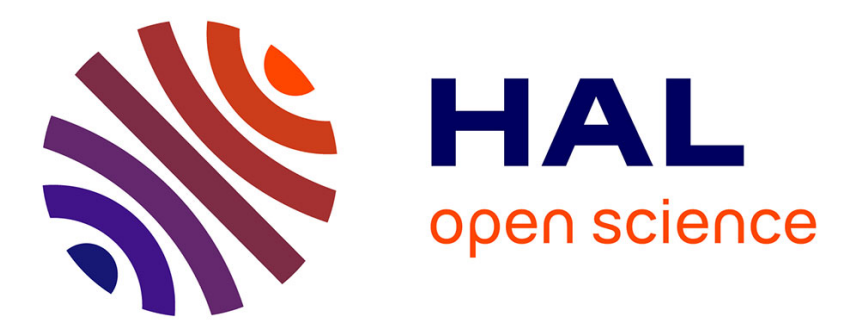

\title{
Torque magnetometry of perpendicular anisotropy exchange-spring heterostructures
}

P Vallobra, Thomas Hauet, F Montaigne, E Shipton, Eric E. Fullerton, S Mangin

\section{- To cite this version:}

P Vallobra, Thomas Hauet, F Montaigne, E Shipton, Eric E. Fullerton, et al.. Torque magnetometry of perpendicular anisotropy exchange-spring heterostructures. Journal of Applied Physics, 2016, 120 (1), pp.13903-13920. 10.1063/1.4955041 . hal-01345416

\author{
HAL Id: hal-01345416 \\ https://hal.science/hal-01345416
}

Submitted on 13 Jul 2016

HAL is a multi-disciplinary open access archive for the deposit and dissemination of scientific research documents, whether they are published or not. The documents may come from teaching and research institutions in France or abroad, or from public or private research centers.
L'archive ouverte pluridisciplinaire HAL, est destinée au dépôt et à la diffusion de documents scientifiques de niveau recherche, publiés ou non, émanant des établissements d'enseignement et de recherche français ou étrangers, des laboratoires publics ou privés. 


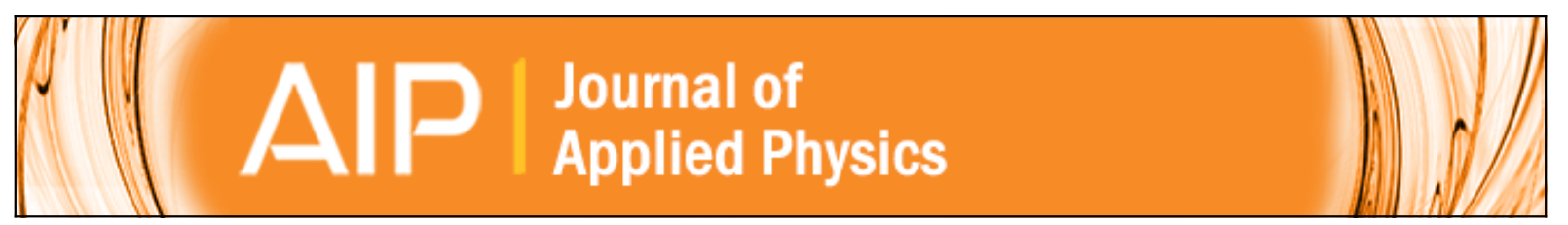

\section{Torque magnetometry of perpendicular anisotropy exchange-spring heterostructures}

P. Vallobra, T. Hauet, F. Montaigne, E. G. Shipton, E. E. Fullerton, and S. Mangin

Citation: Journal of Applied Physics 120, 013903 (2016); doi: 10.1063/1.4955041

View online: http://dx.doi.org/10.1063/1.4955041

View Table of Contents: http://scitation.aip.org/content/aip/journal/jap/120/1?ver=pdfcov

Published by the AIP Publishing

\section{Articles you may be interested in}

Experimental evidence of skyrmion-like configurations in bilayer nanodisks with perpendicular magnetic anisotropy

J. Appl. Phys. 117, 17B529 (2015); 10.1063/1.4918685

Influence of shape, size and internal structure on magnetic properties of core-edge nanodots with perpendicular anisotropy

J. Appl. Phys. 116, 193905 (2014); 10.1063/1.4902169

Variable angle magnetometry for exchange-coupled multilayers with in-plane and perpendicular anisotropy

J. Appl. Phys. 110, 123905 (2011); 10.1063/1.3665191

Crossover from reversible to irreversible magnetic exchange-spring processes in antiferromagnetically exchange-coupled soft/hard bilayer structures

J. Appl. Phys. 108, 043919 (2010); 10.1063/1.3478752

Tunable steady-state domain wall oscillator with perpendicular magnetic anisotropy

Appl. Phys. Lett. 95, 162504 (2009); 10.1063/1.3238314

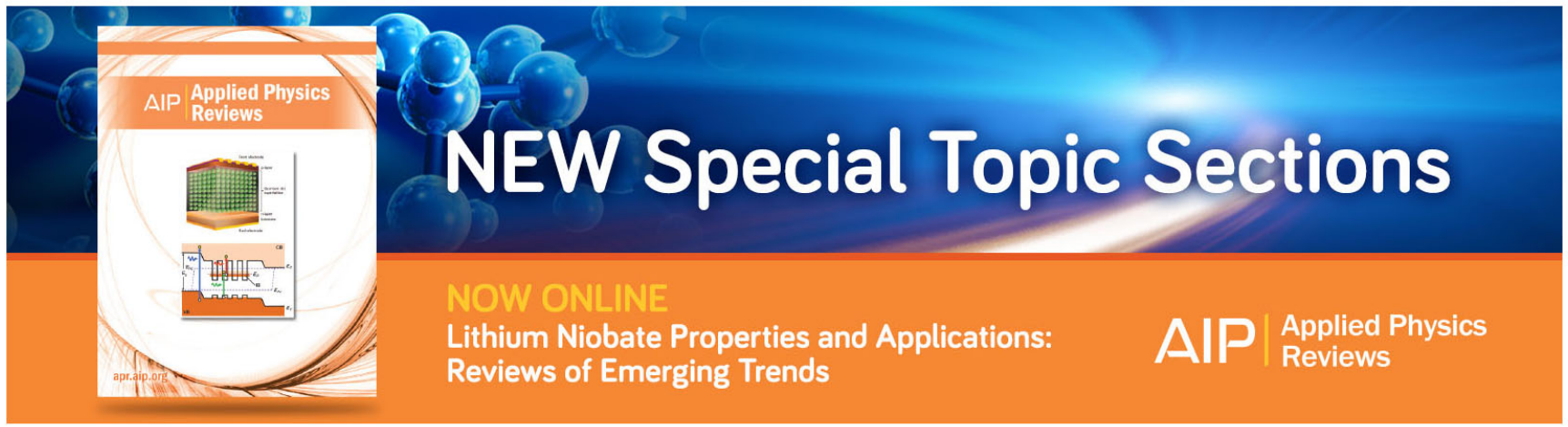




\title{
Torque magnetometry of perpendicular anisotropy exchange-spring heterostructures
}

\author{
P. Vallobra,${ }^{1}$ T. Hauet, ${ }^{1}$ F. Montaigne,${ }^{1}$ E. G. Shipton, ${ }^{2}$ E. E. Fullerton, ${ }^{2}$ and S. Mangin ${ }^{1}$ \\ ${ }^{1}$ Institut Jean Lamour, UMR 7198 CNRS-Université de Lorraine, F-54506 Vandoeuvre lès Nancy, France \\ ${ }^{2}$ Center for Memory and Recording Research, University of California San Diego, La Jolla, \\ California 92093-0401, USA
}

(Received 20 April 2016; accepted 18 June 2016; published online 7 July 2016)

\begin{abstract}
The field-induced magnetic configurations in a $[\mathrm{Co} / \mathrm{Pd}]_{15} / \mathrm{TbFeCo}$ exchange-spring system with perpendicular magnetic anisotropy are studied using torque magnetometry. The experimental results are compared to a 1D micromagnetic simulation. The good agreement between experiments and simulations allows us to deduce the evolution of the in-depth magnetic configuration as a function of the applied field orientation and amplitude. The chirality transition of the interfacial domain wall developing in the structure can also be determined with this technique. Published by AIP Publishing.

[http://dx.doi.org/10.1063/1.4955041]
\end{abstract}

\section{INTRODUCTION}

Torque magnetometer is a technique often used to quantify fundamental magnetic properties such as the magnetic anisotropy. These measurements are mainly performed on single domain samples. ${ }^{1-3}$ However, recently it has been used to study magnetic phase transitions, ${ }^{4}$ exchange biasing, ${ }^{5-7}$ and non-uniform magnetization reversal. ${ }^{8-10}$ Here, we use torque magnetometry to study a bilayer structure in which a lateral interfacial domain wall can be created and controlled. ${ }^{11-15}$ This class of structures has broad applications in areas such as magnetic recording media for hard disk drives ${ }^{16,17}$ permanent magnets, ${ }^{18}$ and spin-transfer-torque Magnetic Random Acess Memory (MRAM) to allow lower switching current while maintaining thermal stability. ${ }^{19}$ Here, we study an anti-ferromagnetically exchange coupled spring system, with perpendicular anisotropy $\mathrm{Tb}_{45}\left(\mathrm{Fe}_{80} \mathrm{Co}_{20}\right)_{55}(24.5 \mathrm{~nm}) /[\mathrm{Co}(0.35 \mathrm{~nm}) / \mathrm{Pd} \quad(0.7)]_{15}$. The sample has been previously characterized using superconducting quantum interference device (SQUID) magnetometry and polarized neutron reflectometry. ${ }^{11}$

The $\mathrm{TbFeCo}$ alloy is a ferrimagnet with the $\mathrm{Tb}$ and $\mathrm{FeCo}$ moments coupled antiferromagnetically. For the alloy composition at room temperature, the $\mathrm{Tb}$ moment prevails and tends to align with an external magnetic field. The $\mathrm{Co} / \mathrm{Pd}$ multilayer is coupled ferromagnetically to the FeCo sublattice and antiferromagnetically to the $\mathrm{Tb}$ sublattice so the $\mathrm{Co} / \mathrm{Pd}$ multilayer is antiferromagnetically coupled to net magnetization of the $\mathrm{TbFeCo}$ layer. Figure 1 shows the room-temperature magnetic hysteresis loop with the field perpendicular to the layers. At remanence, the net magnetization of $[\mathrm{Co} / \mathrm{Pd}]$ and $\mathrm{TbFeCo}$ are anti-parallel to each other as shown in Fig. 2. However, for field amplitudes larger than $0.2 \mathrm{~T}$, the competition between the interfacial anti-ferromagnetic exchange coupling and the external magnetic field leads to the formation of an interfacial domain wall $(i D W)$ whose structure depends on the external field amplitude (Fig. 2). Similar iDWs may be created in bilayers system with both in- and out-of-plane anisotropy. ${ }^{9,11,13-15}$ However, such $i D W \mathrm{~s}$ are difficult to probe only by means of magnetization versus field measurements. The magnetization variation induced by the $i D W$ compression under field may be small compared to the total signal making it difficult to obtain accurate information by fitting the small magnetization variation using a one-dimensional spin chain. Finally, this type of measurements does not give any information on the chirality of the $i D W$.

We previously used polarized neutrons to quantify the $i D W$ as shown in Ref. 11, but we demonstrate here that a much simpler lab technique can give comparable accuracy and valuable information in such coupled systems. Here, the good agreement between torque data and neutron experiments from Ref. 11 demonstrates that torque magnetometer is efficient to characterize complex magnetic structures such as $i D W$. Moreover, torque results can easily be fitted using a 1D-spins chain model. Our results show that while rotating the applied field reversible magnetization rotation can be observed but also irreversible switching corresponding either to a transition from a parallel alignment to an antiparallel

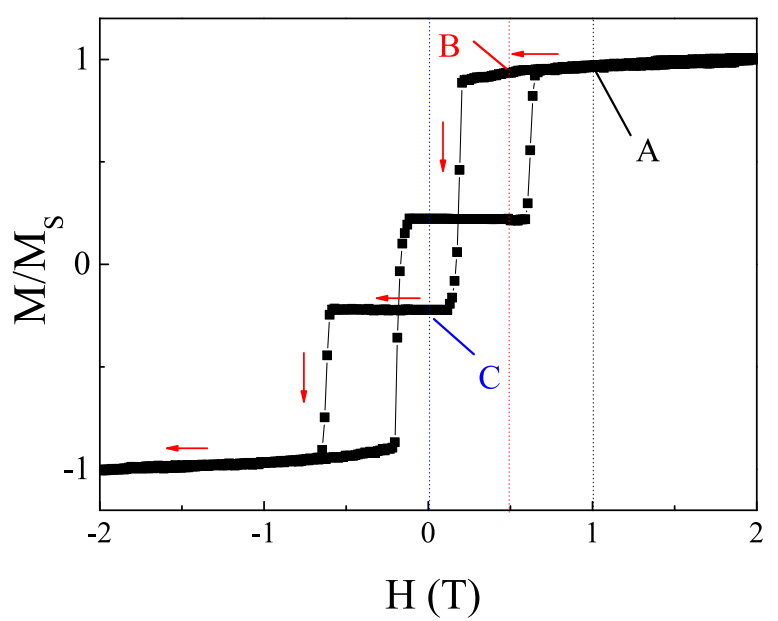

FIG. 1. Normalized magnetization as a function of the magnetic field applied perpendicularly to the film plane for an anti-ferromagnetically exchanged coupled bilayer: $\mathrm{Tb}_{45}\left(\mathrm{Fe}_{80} \mathrm{Co}_{20}\right)_{55}(24.5 \mathrm{~nm}) /[\mathrm{Co}(0.35 \mathrm{~nm}) / \mathrm{Pd}$ $(0.7)]_{15}$. The red arrows help to follow the hysteresis loop evolution from large positive fields to large negative fields. The magnetic configurations of the states indicated by a letter A, B, and C are plotted in Fig. 2. 


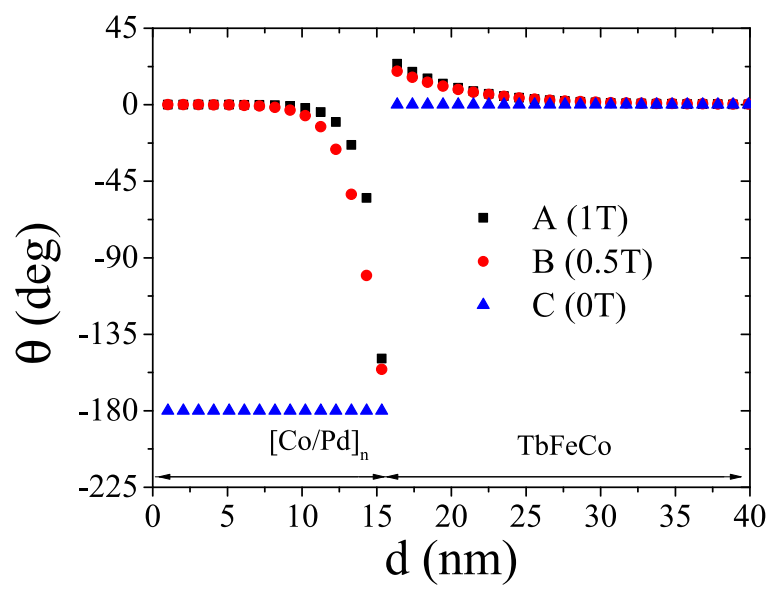

FIG. 2. Magnetic depth profile: Evolution of the angle $(\alpha)$ between the equilibrium magnetization and the direction perpendicular to the film plane as a function of the film depth $\mathrm{d}$ for an applied magnetic field intensity of (a) $1 \mathrm{~T}$, (b) $0.5 \mathrm{~T}$, and (c) $0 \mathrm{~T}$ obtained from a $1 \mathrm{D}$-spin chain model.

alignment, or to a transition between the two antiparallel or the two parallel alignments and/or to chirality transitions.

\section{EXPERIMENTAL DETAILS}

$\mathrm{Tb}_{45}\left(\mathrm{Fe}_{80} \mathrm{Co}_{20}\right)_{55} \quad(24.5 \mathrm{~nm}) /[\mathrm{Co} \quad(0.35 \mathrm{~nm} \quad \mathrm{~nm}) / \mathrm{Pd}$ $(0.7 \mathrm{~nm})] \mathrm{x}_{15} / \mathrm{Pd}(2 \mathrm{~nm})$ multilayers were grown at room temperature onto Si wafers by dc magnetron sputtering. All deposition rates were previously calibrated by fitting low angle $\mathrm{X}$-ray reflectivity measurements. The ferrimagnetic $\mathrm{TbFeCo}$ alloy exhibits a strong perpendicular magnetic anisotropy (PMA) and for this composition the net magnetization of the alloy is parallel to the $\mathrm{Tb}$ moments and antiparallel to the $\mathrm{Fe}$ and Co moments. $[\mathrm{Co} / \mathrm{Pd}]$ multilayers show PMA and the $\mathrm{Pd}$ thickness determines the exchange coupling between successive Co layers and determines the effective exchange in the $\mathrm{Co} / \mathrm{Pd}$ layer.

Torque magnetometry curves were obtained using a commercial PPMS torque magnetometer at $300 \mathrm{~K}$ for fields ranging from 0 to $7 \mathrm{~T}$. The angle between the direction of the magnetic field and the anisotropy axis of the sample is given by $\alpha$. For each set of data, the same procedure is used. First a magnetic field, set at a fixed amplitude $(0.5,1$, and $5.5 \mathrm{~T}$ ), is applied perpendicularly to the sample plane (angle $\alpha=0^{\circ}$ ). Then $\alpha$ is monotonically changed to reach $360^{\circ}$ and then reduced back to $0^{\circ}$.

The experimental evolution of the torque curve is compared to results obtained from the micromagnetic 1D-spin chain model described in Refs. 11, 12, and 15. The torque curve is calculated as the derivative of the energy relatively to the angle $\alpha .^{7}$ The total energy is calculated by assuming a chain of 40 magnetic moments each describing an approximately $1 \mathrm{~nm}$ layer thick. The magnetic energy of the multilayer per area unit is calculated by considering the exchange energy, the anisotropy energy, the Zeeman energy corresponding to the interaction of the magnetization with the applied field $\mathrm{H}$, and finally the interface exchange energy (characterized by a negative exchange stiffness A).

The following parameters were used consistently with Ref. 11: $M_{\mathrm{TbFeCo}}=320 \mathrm{kA} / \mathrm{m}, M_{\mathrm{Co} / \mathrm{Pd}}=710 \mathrm{kA} / \mathrm{m}$, the anisotropy constant $H_{\mathrm{K}} \mathrm{TbFeCo}=20 \mathrm{kOe}, H_{\mathrm{K}} \mathrm{Co} / \mathrm{Pd}=10 \mathrm{kOe}$, and the exchange stiffness: $A_{\mathrm{TbFeCo}}=10^{-15} \mathrm{~J} \mathrm{~m}, A_{\mathrm{Co} / \mathrm{Pd}}=10^{-16} \mathrm{~J} \mathrm{~m}$. The interfacial exchange coupling is held constant at $A_{\text {interface }}=-9 \times 10^{-16} \mathrm{~J} \mathrm{~m}$. Starting from four initial magnetic configurations in which the net magnetizations are along the easy axis (two for which the magnetizations of the two layers are parallel and two for which the magnetizations of the two layers are anti-parallel), the energy is minimized in order to determine the possible magnetic metastable and stable states. Those states are noted respectively $S_{1}, S_{2}, S_{3}$, and $\mathrm{S}_{4}$.

\section{RESULTS AND DISCUSSION}

Shown in Fig. 3 are the experimental torque curves that are compared with the calculated solutions for both various metastable states and the ground state depending on the angle $\alpha$. Black curves correspond to the expected torque curve if the system could always minimize its energy to reach the ground state. The first feature to highlight is that the experimental curves are well reproduced by the calculations and as the field rotates there are hysteretic jumps between the ground state and metastable states. The model does not predict the field for these hysteretic transitions which likely occurs by nucleation and domain growth. In the case of a $5.5 \mathrm{~T}$ field, the moments of the $[\mathrm{Co} / \mathrm{Pd}] / \mathrm{TbFeCo}$ bilayer are close to being aligned with the field for all $\alpha$ and the curves reflect the uniaxial anisotropy of the system. The $i D W$ width is estimated to be close to $5 \mathrm{~nm}$. The existence of a very thin remaining $i D W$ is only marked by the discontinuity around $\alpha=180^{\circ}$ which also appears in the ground state curve. The increasing $\alpha$ branch and the decreasing $\alpha$ branch are mostly superposed since the magnetization rotation is here nearly reversible. The irreversible jump around $180^{\circ}$ is due to the transition from one domain wall chirality to the other. This transition can be observed for the three field values $(0.5$, 1 , and $5.5 \mathrm{~T}$ ) and was previously observed for in plane magnetized system. ${ }^{9}$ The intrinsic chirality (clockwise or anticlockwise) of the domain wall is determined at low angles by the sense of rotation of the field and evolves due the chirality's transitions. This can be verified by the described 1D simulation.

In Fig. 4, we present the evolution of the magnetic energy and the magnetic configurations when crossing the transition around $180^{\circ}$ under $1 \mathrm{~T}$ field (Fig. 3(b)). The evolution of the energy of the two possible magnetic states are shown as a function of $\alpha$ (Fig. 4(a)), whereas the magnetic configurations of those two states for $\alpha=170^{\circ}$ and $\alpha=190^{\circ}$ are shown in Fig. 4(b). One can clearly observe that the hysteretic transition around $\alpha=180^{\circ}$ is a chirality transition. Note that in our simulation the moments inside the $i D W$ can only rotate in one plane. While our results are consistent with a Neel-like wall, they do not exclude the possibility of more complex DWs with in-plane and out-of-plane components.

Hysteretic behavior can also be observed around $90^{\circ}$ and $270^{\circ}$ as presented in Figs. 3(a) and 3(b) for applied field of 0.5 and $1 \mathrm{~T}$. To illustrate the change in the magnetic configurations at this transition, we have plotted the possible 


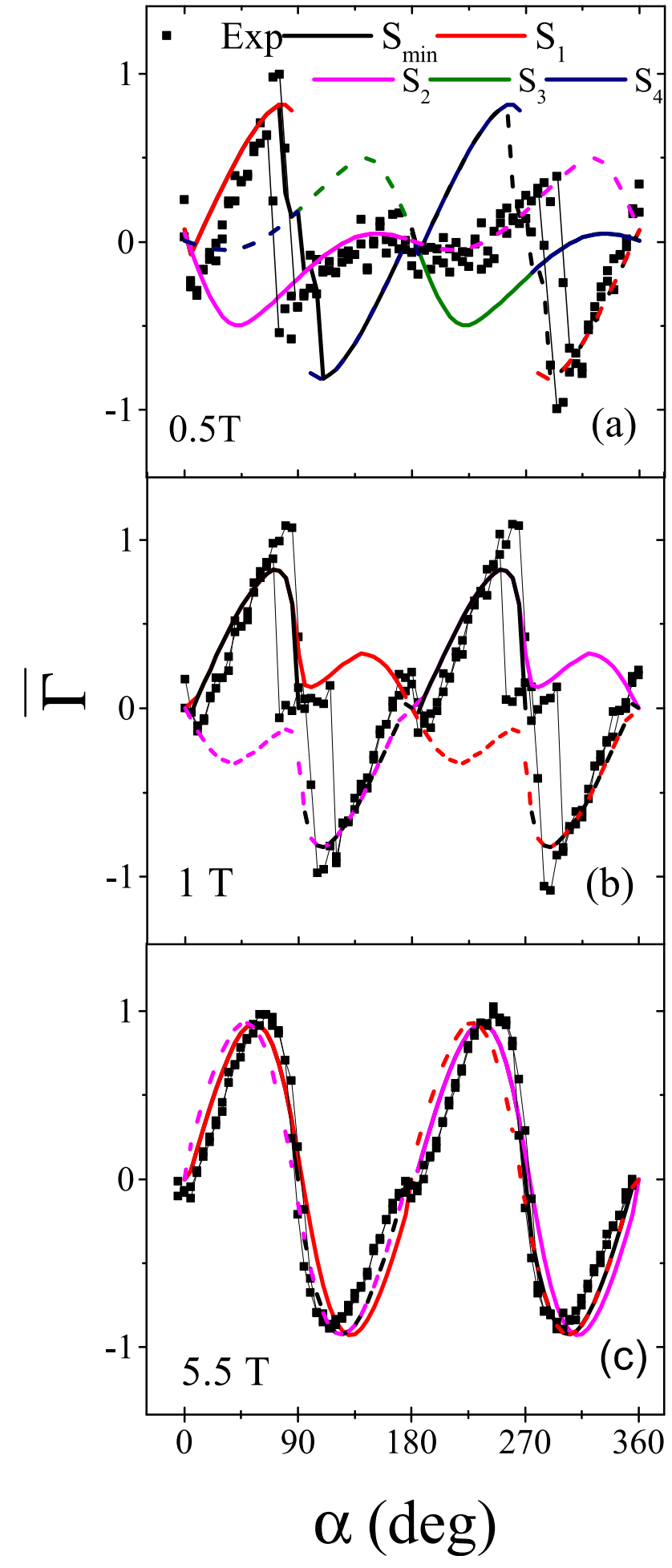

FIG. 3. Evolution of the normalized torque as a function of the field angle $\alpha$ measured at $300 \mathrm{~K}$ under various magnetic fields amplitude $0.5 \mathrm{~T}$ (a), $1 \mathrm{~T}$ (b), and $5.5 \mathrm{~T}$ (c). Comparison between experimental torque measurements (green stars) and the solutions $\left(\mathrm{S}_{1}, \mathrm{~S}_{2}, \mathrm{~S}_{3}\right.$, and $\left.\mathrm{S}_{4}\right)$ of a $1 \mathrm{D}$ simulation is shown. For $\mathrm{S}_{1}, \mathrm{~S}_{2}, \mathrm{~S}_{3}$, and $\mathrm{S}_{4}$ when the curve is in dashed the chirality of the $i D W$ is anticlockwise and is in solid when the chirality of the $i D W$ is clockwise. The black curve corresponds to the calculated torque curve in the case where the system is in the minimum energy configuration at each angle $\alpha$.

magnetic configurations for $\alpha=80^{\circ}$ and $\alpha=100^{\circ}$ under $0.5 \mathrm{~T}$ in Fig. 5.

In this case, we can identify that at the transition the magnetic configuration changes from a quasi parallel
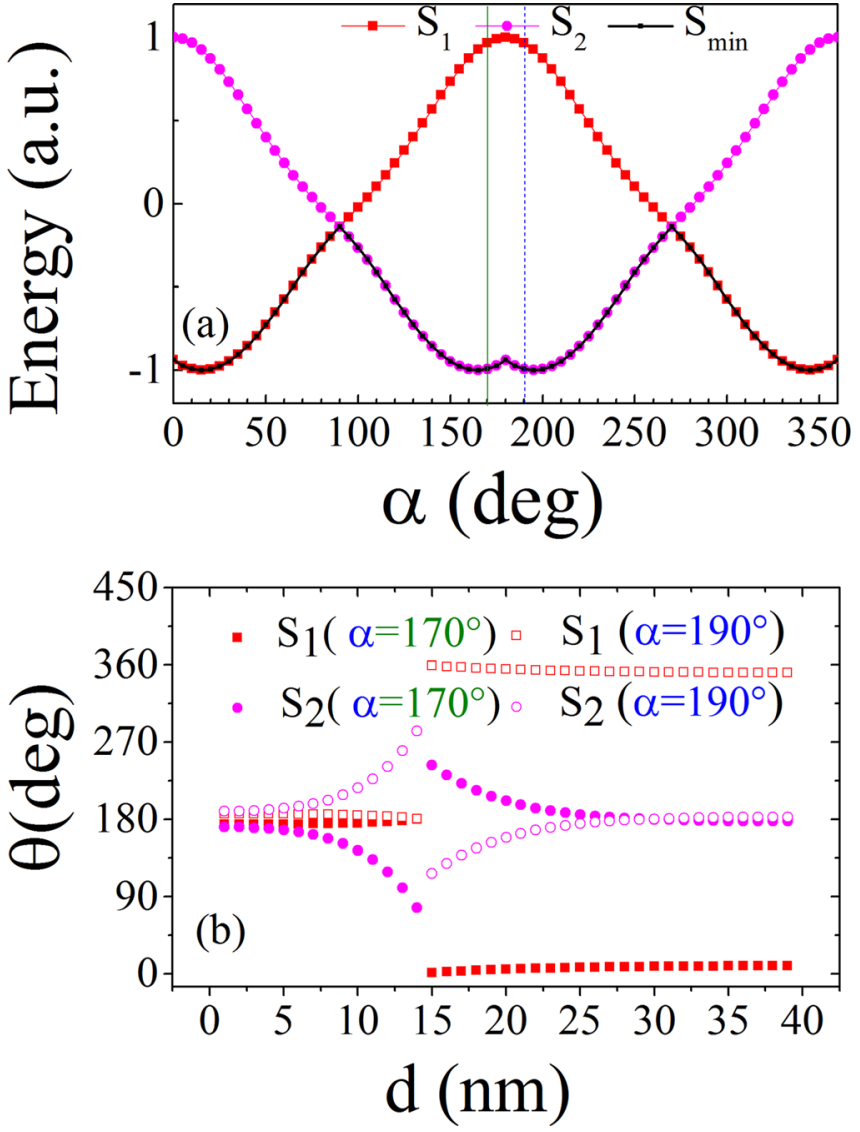

FIG. 4. (a) Evolution of the total energy of the sample as a function of the field angle $\alpha$ for $1 \mathrm{~T}$. The two possible solutions are shown in red and purple. The black curve corresponds to the configuration of minimal energy. (b) Magnetic depth profile: Evolution of moment direction given by $\theta$ as a function of the sample depth for a magnetic field intensity of $1 \mathrm{~T}$ and two orientation $\alpha=170^{\circ}$ and $\alpha=190^{\circ}$.

alignment of the magnetization of the $\mathrm{TbFeCo}$ and the $\mathrm{Co} / \mathrm{Pd}$ layer leading to the formation of an iDW mainly located in the $\mathrm{Co} / \mathrm{Pd}$ multilayer to a quasi-antiparallel alignment of the magnetization where no iDW is present. This transition can also be viewed as reversal of the $\mathrm{TbFeCo}$ magnetization from $0^{\circ}$ to $180^{\circ}$.

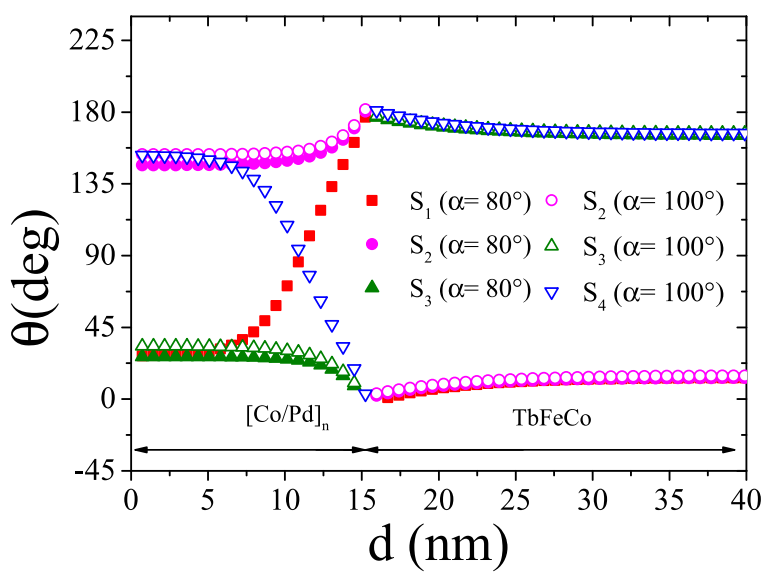

FIG. 5. Magnetic depth profile: Evolution of moment direction given by $\alpha$ as a function of the position along the direction perpendicular to the film plane for a magnetic field intensity of $0.5 \mathrm{~T}$ and different orientation $\alpha=80^{\circ}$ and $\alpha=100^{\circ}$. 


\section{CONCLUSION}

We have shown that field-dependent torque measurement is a powerful experimental method to study the evolution of the magnetic configuration in coupled magnetic systems. In $[\mathrm{Co} / \mathrm{Pd}] / \mathrm{TbFeCo}$ which is an antiferromagnetically exchange coupled bilayer exchange spring system with PMA, the evolution of the interface domain wall has been followed as an applied field is rotating. The chirality transitions of the domain wall could be followed and irreversible change detected.

\section{ACKNOWLEDGMENTS}

This work was supported by the ANR-NSF Project, ANR13-IS04-0008-01, "COMAG" by the ANR-Labcom Project LSTNM, Experiments were performed using equipment from the TUBE_-Daum funded by FEDER (EU), ANR, the Region Lorraine, and Grand Nancy. Work at UCSD was supported by NSF Award No. DMR-1312750.

${ }^{1}$ Y. Henry, K. Ounadjela, L. Piraux, S. Dubois, J.-M. George, and J.-L. Duvail, Eur. Phys. J. B 20, 35-54 (2001).

${ }^{2}$ D. H. Shin, G. Suran, D. Chumakow, R. Schafer, and Y. Henry, J. Appl. Phys. 91, 8237-8239 (2002).

${ }^{3}$ J. Rigue, D. Chrischon, A. M. H. de Andrade, and M. Carara, J. Magn. Magn. Mater. 324, 1561 (2012).
${ }^{4}$ D. G. Naugle, B. I. Belevtsev, K. D. D. Rathnayaka, S.-I. Lee, and S. M. Yeo, J. Appl. Phys. 103, 07B718 (2008).

${ }^{5}$ J. Nogués and I. K. Schuller, J. Magn. Magn. Mater. 192, 203 (1999).

${ }^{6}$ T. Gredig, I. N. Krivorotov, and E. Dan Dahlberg, Phys. Rev. B 74, 094431 (2006).

${ }^{7}$ E. Shipton, K. Chan, T. Hauet, O. Hellwig, and E. E. Fullerton, Appl. Phys. Lett. 95, 132509 (2009).

${ }^{8}$ S. L. Tomarken, A. F. Young, S. W. Lee, R. G. Gordon, and R. C. Ashoori, Phys. Rev. B 90, 201113(R) (2014).

${ }^{9}$ C. L. Platt, A. E. Berkowitz, S. David, E. E. Fullerton, J. S. Jiang, and S. D. Bader, Appl. Phys. Lett. 79, 3992 (2001).

${ }^{10}$ Z. Shi, J. Du, R. W. Chantrell, S. Mangin, and S. M. Zhou, Appl. Phys. Lett. 98, 122507 (2011).

${ }^{11}$ S. M. Watson, T. Hauet, A. Borchers, S. Mangin, and E. E. Fullerton, Appl. Phys. Lett. 92, 202507 (2008).

${ }^{12}$ S. Mangin, L. Thomas, F. Montaigne, W. Lin, T. Hauet, and Y. Henry, Phys. Rev. B 80, 224424 (2009).

${ }^{13}$ J. McCord, Y. Henry, T. Hauet, F. Montaigne, E. E. Fullerton, and S. Mangin, Phys. Rev. B 78, 094417 (2008).

${ }^{14}$ S. Mangin, F. Montaigne, and A. Schuhl, Phys. Rev. B 68, 140404(R) (2003).

${ }^{15}$ F. Montaigne, S. Mangin, and Y. Henry, Phys. Rev. B 67, 144412 (2003).

${ }^{16}$ A. Berger, N. Supper, Y. Ikeda, B. Lengsfield, A. Moser, and E. E. Fullerton, Appl. Phys. Lett. 93, 122502 (2008).

${ }^{17}$ E. E. Fullerton, H. Do, D. T. Margulies, and N. F. L. Super, U.S. patent 7,425,377 (16 September 2008).

${ }^{18}$ H. Zeng, J. Li, J. P. Liu, Z. L. Wang, and S. Sun, Nature 420(6914), 395-398 (2002).

${ }^{19}$ I. Yulaev, M. V. Lubarda, S. Mangin, V. Lomakin, and E. E. Fullerton, Appl. Phys. Lett. 99, 132502 (2011). 